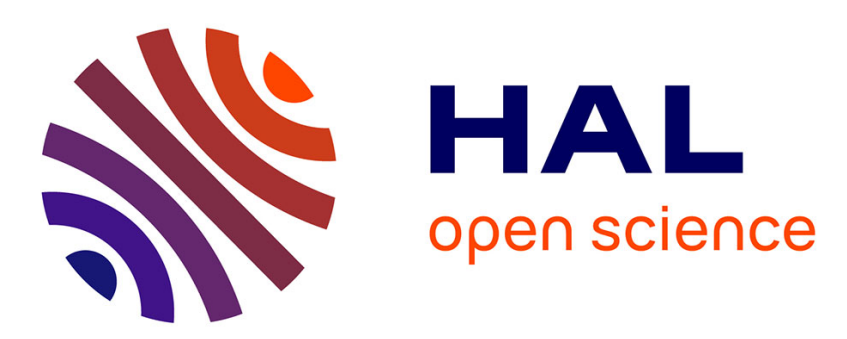

\title{
Avalanche mobility induced by the presence of an erodible bed and associated entrainment
}

Anne Mangeney, L. S. Tsimring, D Volfson, Igor S. Aranson, François Bouchut

\section{To cite this version:}

Anne Mangeney, L. S. Tsimring, D Volfson, Igor S. Aranson, François Bouchut. Avalanche mobility induced by the presence of an erodible bed and associated entrainment. Geophysical Research Letters, 2007, 34, pp.22401. 10.1029/2007GL031348 . insu-01285125

\section{HAL Id: insu-01285125 \\ https://hal-insu.archives-ouvertes.fr/insu-01285125}

Submitted on 8 Mar 2016

HAL is a multi-disciplinary open access archive for the deposit and dissemination of scientific research documents, whether they are published or not. The documents may come from teaching and research institutions in France or abroad, or from public or private research centers.
L'archive ouverte pluridisciplinaire HAL, est destinée au dépôt et à la diffusion de documents scientifiques de niveau recherche, publiés ou non, émanant des établissements d'enseignement et de recherche français ou étrangers, des laboratoires publics ou privés. 


\title{
Avalanche mobility induced by the presence of an erodible bed and associated entrainment
}

\author{
A. Mangeney, ${ }^{1,2}$ L. S. Tsimring, ${ }^{1}$ D. Volfson, ${ }^{1}$ I. S. Aranson, ${ }^{3}$ and F. Bouchut ${ }^{4}$ \\ Received 16 July 2007; revised 14 September 2007; accepted 9 October 2007; published 20 November 2007.
}

[1] The partial fluidization model developed by Aranson and Tsimring (2002) is used to simulate the spreading of a 2D circular cap of granular material over an erodible bed made of the same material. Numerical results show that the presence of even a very thin layer of granular material lying on the solid bed strongly increases the mobility of granular flows. Furthermore, as the thickness of the granular layer increases, the dynamics of the flowing mass changes from a decelerating avalanche to a traveling wave. Numerical simulation suggest that surges are generated if enough mass is entrained, increasing the energy of the flowing material and balancing the energy lost by friction. Citation: Mangeney, A., L. S. Tsimring, D. Volfson, I. S. Aranson, and F. Bouchut (2007), Avalanche mobility induced by the presence of an erodible bed and associated entrainment, Geophys. Res. Lett., 34, L22401, doi:10.1029/2007GL031348.

\section{Introduction}

[2] Dense granular flows driven by gravity, such as landslides or avalanches, participate in the evolution of the surface of the Earth and other telluric planets. They also represent natural hazards that are a threat to many populations and infrastructures. However, natural granular flows are still poorly understood. In particular, the origin of the high mobility of avalanches or the occurrence of surges during their propagation along the slope are challenging questions. Numerical models as well as laboratory experiments on granular flows have been able to reproduce several geomorphological features or scaling laws observed in terrestrial or Martian avalanches [see, e.g., Pudasaini and Hutter, 2007; Mangeney et al., 2007]. However when compared quantitatively to field observations, experimental as well as numerical results fail to explain the high mobility of geophysical gravitational flows that can travel very long distances even on small slopes [Davies and McSaveney, 1999; Lucas and Mangeney, 2007].

[3] Fundamentally different mechanisms were employed to explain the high mobility of gravitational flows, e.g., upward current of air, hovercraft action at the base, melting of rock, fluidization induced by the presence of fine dust, acoustic fluidization, frictional heat, hydroplanning, grains fragmentation, polydispersity or the potential presence of

\footnotetext{
${ }^{1}$ Institute for Nonlinear Science, University of California, San Diego, La Jolla, California, USA.

${ }^{2}$ Institut de Physique du Globe de Paris, University Paris 7, Paris, France.

${ }^{3}$ Argonne National Laboratory, Argonne, Illinois, USA.

${ }^{4}$ Département de Mathématiques et Applications, Ecole Normale Superieure, Paris, France.
}

Copyright 2007 by the American Geophysical Union. 0094-8276/07/2007GL031348 water or air within the granular mass (see Legros [2002] and Pudasaini and Hutter [2007] for reviews).

[4] Among these processes, the erosion of material already present on the underlying solid topography is expected to play a significant role in the mobility of debris or snow avalanches and overall dynamics of transportation [McDougall and Hungr, 2005; Sovilla et al., 2006]. In geological context, the entrainment of the substrate by the flowing mass could either accelerate or decelerate the flow (i.e., increase or decrease its mobility) depending on the nature and on the consolidation of the erodible material as well as on the topography of the bedrock and on the dynamics of the flow.

[5] When static granular material is entrained into motion by the flowing material, no-flow and flowing zones not only coexist but exchange mass and momentum. A theoretical description of these processes is still lacking even for much simpler configurations involving dry granular flows in laboratory experiments. In the case of the spreading of a three-dimensional granular cap released on an inclined bed, Pouliquen and Forterre [2002] show that the presence of a shallow erodible bed build-up of the same granular material induces triangular shaped traveling waves propagating at a constant velocity downslope. Triangular shaped quasi-onedimensional waves have also been observed and studied theoretically when a perturbation is imposed at the top of a thin granular layer over an inclined plane [Mallogi et al., 2006, Aranson et al., 2006]. However, no systematic studies of the transition between granular flows leaving a deposit on the slope and the generation of traveling waves have been performed.

[6] Classical depth-averaged thin layer models fail in simulating triangular shaped waves traveling over an erodible bed when entrainment is not taken into account [Pouliquen and Forterre, 2002]. When introduced in numerical depth-averaged models, entrainment is generally approximated on the basis of questionable phenomenological laws or closure relations [Bouchut et al., 2007]. Furthermore, this approach is only valid when the granular material is well divided into a thin surface flow and a static layer. However, in many important situations, the granular material remains in a multiphase state when part of it is fluidized while another part is solid.

[7] Aranson and Tsimring [2002, 2006] introduced an approach that does not involve depth-averaging, and describes explicitly the static/flowing transition through an order parameter related to the fraction of static contacts between the grains. This partial fluidization model has been shown to be able to reproduce a large spectrum of experimental results [e.g., Aranson et al., 2006] and has been calibrated with molecular dynamics simulations [Volfson et al., 2003]. 

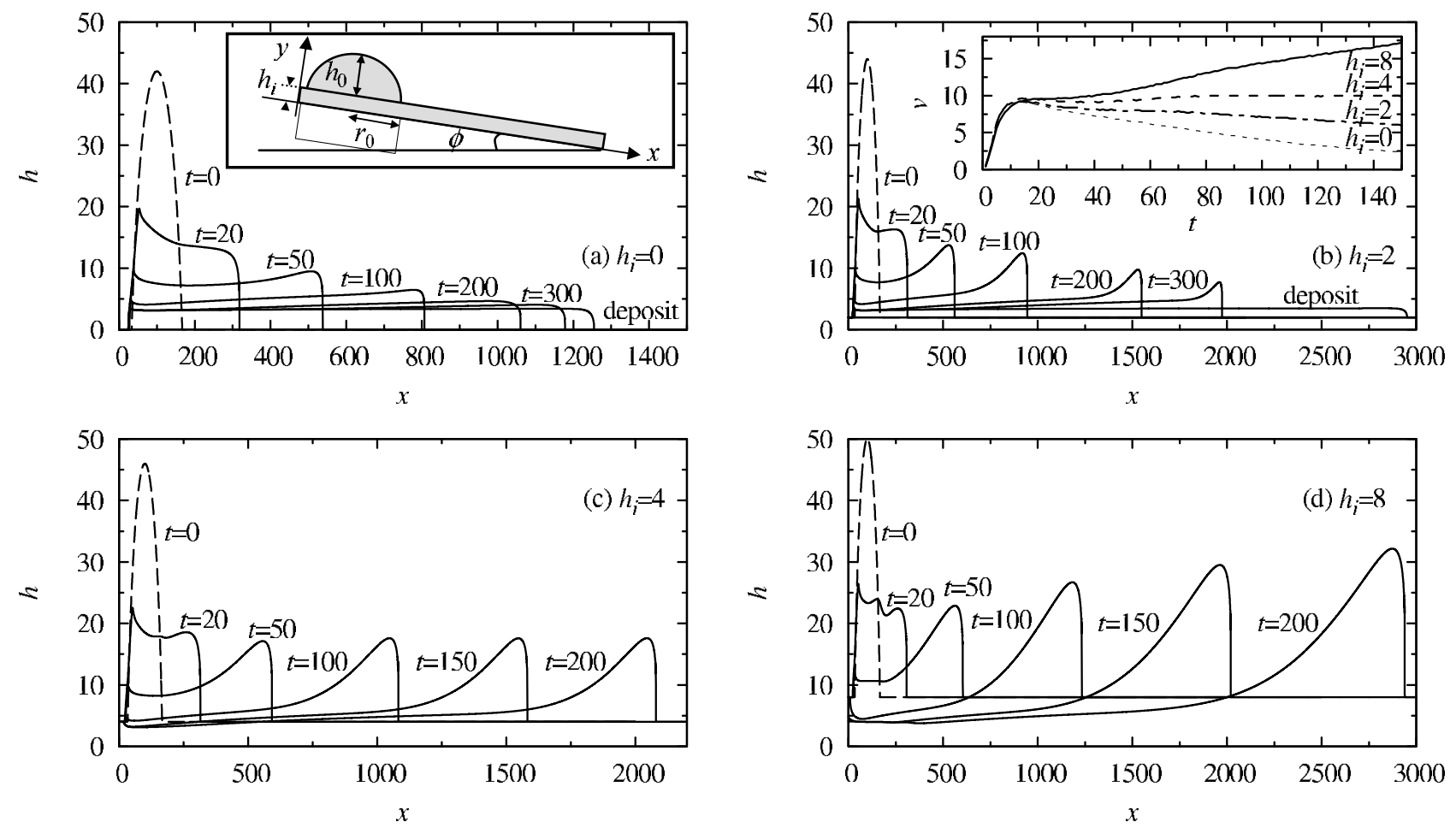

Figure 1. Thickness $h$ as a function of the down slope position $x$ at several times for the granular flow on (a) a rigid bed $h_{i}=0$ and (b-d) a plane covered by an initial layer of material $h_{i}=2,4$ and 8 , respectively. The initial profile is represented in dashed lines. For decelerating avalanches, the granular mass stops at (a) $t=560$, and (b) $t=930$. Inset in Figure 1a shows schematic figure of the numerical setup. Inset in Figure $1 \mathrm{~b}$ shows front velocity $v$ of the granular mass as a function of time for $h_{i}=0,2,4$ and 8 .

[8] Using the partial fluidization model, we show here that the presence of even a very thin layer of static granular material lying on the topography strongly increases the mobility of granular flows made of the same material. In the range of material and slope considered here, the dynamics of the granular flow changes from a decelerating avalanche leading to a deposit on the slope to a traveling wave propagating downslope as the thickness of the erodible bed layer increases.

\section{Numerical Setup and Model}

[9] The transition between decelerating avalanches leading to a deposit on the slope and traveling waves propagating downslope is studied here by simulating the spreading of a two-dimensional granular cap on an inclined plane covered by a shallow erodible layer with variable thickness $h_{i}$ (see inset in Figure 1a). Although the geometrical parameters have been chosen to simulate the spreading of the small cap in the experiments of Pouliquen and Forterre [2002], the 2D numerical results may be compared only qualitatively to the $3 \mathrm{D}$ experimental results. The shape of the cap is a circular segment with dimensionless radius $r_{0}=$ 66.5 and thickness $h_{0}=42$, where all the space variables have been scaled by the typical diameter of the particles $d=$ $0.5 \mathrm{~mm}$. This cap, centered at the downslope position $x=$ 100 , is released from rest on a plane $x \in[0,3500]$ with inclination $\phi=23^{\circ}$. The bed is covered by a thin static layer of grains of thickness $h_{i}=0,2,4$, and $8\left(h_{i} \ll h_{0}\right)$.
[10] The partial fluidization model is based on the fact that, in slow dense flows, some of the grains are sliding past each other, while others maintain prolonged static contacts with neighbors due to friction and jamming [Aranson and Tsimring, 2002]. The stress tensor is then written as a sum of the fluid part $\sigma_{i j}^{f}$ proportional to the flow strain rate $\dot{\gamma}_{i j}$ and the strain-rate independent part $\sigma_{i j}^{s}$ as it is usually performed [see, e.g., Johnson and Jackson, 1987]

$$
\sigma_{i j}=\sigma_{i j}^{f}+\sigma_{i j}^{s},
$$

with $i=x, y, x$ and $y$ being the direction along the slope and normal to the bed, respectively. Let us call $q$ the ratio between the fluid stress and the total shear stress

$$
\sigma_{x y}^{f}=q \sigma_{x y} .
$$

[11] Correspondingly,

$$
\sigma_{x y}^{s}=[1-q] \sigma_{x y} .
$$

[12] By using discrete element simulation of shear granular layers and calculating the stress tensors and the number of contacts between the grains, Volfson et al. [2003] show that the function $q$ is empirically related to the order 
parameter (OP) $\rho$, or the fraction of static contacts between the grains to the total number of contacts, via the relation $q(\rho)=(1-\rho)^{2.7}$.

[13] The OP $\rho$ is a function of space and time, and it varies continuously from $\rho=0$ in the liquid phase to $\rho=1$ in the solid phase. Since we treat the fluidization of granular layer as a phase transition, we describe the OP by the partial differential equation well known in the theory of phase transitions (time-dependent Ginzburg-Landau equation [Landau and Lifshitz, 1980])

$$
\frac{\partial \rho}{\partial t}+\mathbf{u} \cdot \nabla \rho=\nabla^{2} \rho-f(\rho, \delta)
$$

[14] Here $t$ is time, and $\mathbf{u}$ is the velocity normalized by the time unit $\tau=\sqrt{d / g}$ and the characteristic velocity $\sqrt{g d}$ respectively, where $g$ is the acceleration due to gravity. The fluidization (i.e., the evolution of $\rho$ ) is governed by a control parameter $\delta$ that is defined as the ratio of the (local) shear stress to the normal stress in the spirit of the Mohr-Colomb yield criterion. The OP $\rho$ is advected by the flow (second term in the 1.h.s.) and spreads by diffusion (first term in the r.h.s.). The dynamics of the OP is controlled by the source term $f$ which has been calibrated based on discrete element simulations (see details in Volfson et al. [2003]). The empirical function $f(\rho, \delta)$ has been fitted so that the solid state $(\rho=1)$, the fluid state $(\rho=0)$ and a partially fluidized state $(\rho<1)$ are three possible steady uniform solutions of equation (4) [Aranson and Tsimring, 2002]. Without dwelling on the details of the theory [see Aranson and Tsimring, 2002], we note that this model describes a hysteretic (first-order) transition from a state in which $\rho=1$ to a partially fluidized state in which $\rho<1$ as the control parameter $\delta$ is increased. Unlike the classical MohrColomb picture, due to the hysteresis the GinzburgLandau model yields two critical values of $\delta$ limiting the range of $\delta$ where two phases (solid and fluidized) coexist. The critical values of $\delta$ are directly related to static and dynamic repose angles and depend on the material properties of the granular media including its roughness, polydispersity, friction between the grains, etc. We specified the details of the order parameter model on the basis of detailed molecular dynamics simulations of shear granular layers in Aranson and Tsimring [2002] and Volfson et al. [2003]. While the OP is obviously related to the density, the relative changes of density for dense granular flows are typically very small and the incompressibility approximation holds. However, the static/flowing transition occurring for example at the onset of the flow is known to depend on small decrease in density (dilatancy).

[15] The OP dynamics is coupled to the classical hydrodynamic equations via (2). Inertia has been shown to be small in the flow regime investigated here [Pouliquen and Forterre, 2002] and has been neglected although it is generally significant when dealing with real flows over steep slopes. Furthermore, the granular layer is considered to be thin: the $x$-derivative of the shear stress $\sigma_{x y, x}$ is neglected in the vertical momentum equation as well as the $x$-derivative of the vertical velocity $u_{y, x}$ in the shear strain rate: $\dot{\gamma}_{x y}=u_{x, y}$, where $u$ is normalized velocity. The force balance in the bulk of the thin granular layer then reads

$$
\sigma_{x x, x}+\sigma_{x y, y}=-\sin \phi, \sigma_{y y, y}=\cos \phi,
$$

where the stress tensor is normalized by $\rho_{0} g d$ with $\rho_{0}$ the density of the granular material. The vertical equilibrium leads to the hydrodynamic pressure $\sigma_{y y}=-\cos \phi(h-y)$, where $h(x)$ is the thickness of the granular layer. The horizontal momentum conservation in equation (5) allows to calculate the total shear stress for the flow over an inclined plane $\left(\partial_{x} \phi=0\right)$ when isotropy of normal stress is assumed $\sigma_{x y}=\cos \phi\left(\tan \phi-\partial_{x} h\right)(h-y)$. Note that introducing anisotropy of normal stresses in depth-averaged model to simulate this experiment gives less accurate results than those obtained by assuming isotropy of normal stresses [Pouliquen and Forterre, 2002]. We assume the usual form for the stress-strain rate relation for dense granular flows in the fluid phase: $\sigma_{x y}^{f} \approx \mu \dot{\gamma}_{x y}$ where the viscosity of fluid phase $\mu$ is function of pressure $p=-\sigma_{y y}, \mu=\mu_{0} \sqrt{p}, \mu_{0}=$ const [Da Cruz et al., 2005; I. S. Aranson et al., Rheological properties of partially fluidized granular flows, manuscript in preparation, 2007]. The solid part of the stress tensor is related to the jamming and Coulomb friction of granular particles, and is specified via the order parameter and the full stress, see (3).

[16] Using equation (2) and the stress-stain relation, the horizontal velocity is calculated by integrating the following relation $u_{x, y}=q(\rho) \sigma_{x y} / \sqrt{p} \mu_{0}$. We assume no slide at the base of the flow, i.e., $u(x, y=0)=0$, an assumption that may not be satisfied for some geological flows. Finally, the change in time of the thickness is calculated using mass conservation $\partial_{t} h+\nabla h \bar{u}=0$, where $\bar{u}$ is the depth-averaged horizontal velocity. An upwind scheme suited to deal with shock propagation is used to solve numerically the hyperbolic mass conservation equation.

[17] In the following simulations, the granular layer is initially static ( $\rho=1$ for $h \leq h_{i}$ ) and the granular cap is assumed to be slightly fluidized $(\rho=0.99)$. The boundary conditions are $\rho(x, y=0)=1$ corresponding to a static state at the base and zero flux at the free surface, $\partial_{y} \rho(x, y=h)=0$ is imposed. The values of the parameters in the phenomenological OP model [see Volfson et al., 2003] have been calibrated to roughly reproduce the experiments $\left(\mu_{0}=\right.$ 0.1). Additional simulations show that the results are qualitatively similar when varying these parameters. However a detailed comparison with 2D experimental results have to be done to fit better the parameters making it possible to get quantitative results.

\section{Impact of Erosion on Avalanche Mobility}

[18] Over a rigid bed, the granular mass rapidly spreads on the inclined plane and forms a rounded front which slowly stretches downhill (Figure 1a). The rear part of the mass stops (at $t \simeq 170$ ) and leaves a nearly flat layer of material $\left(h_{s} \simeq 3.3\right)$ on the plane as the avalanche decelerates, and the system equilibrates. These results are in a very good qualitative agreement with the experiments performed by Pouliquen and Forterre [2002] although a sharper front is observed in the simulation at the beginning of the 


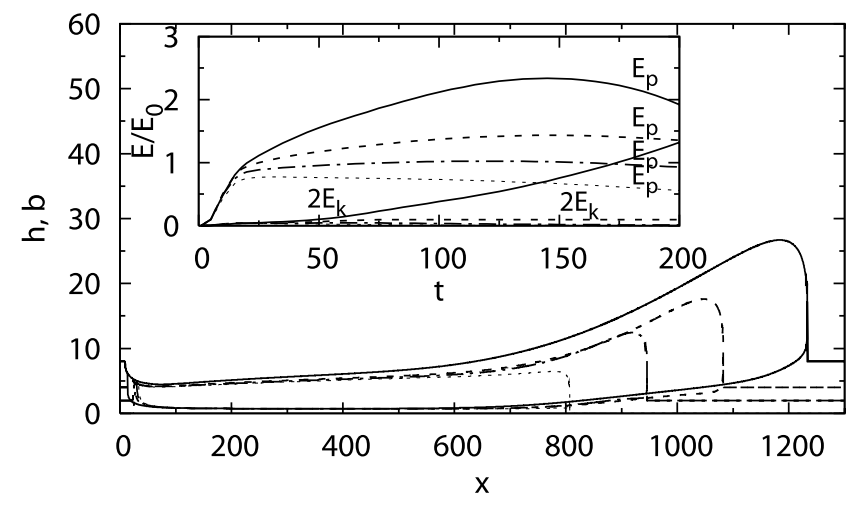

Figure 2. Outline of the fluidized mass defined as a zone where $0<\rho<0.8$, bounded by the free surface and the curve $\rho=0.8$ (this line always exists between the free surface and the bottom $y=0$ where static condition $\rho=1$ is imposed). The fluidized zones are shown at $t=100$ for $h_{i}=$ 0 (dotted line), $h_{i}=2$ (dash-dotted line), $h_{i}=4$ (dashed line), and $h_{i}=8$ (full line). Inset shows potential energy $E_{p}$ and twice the kinetic energy $2 E_{k}$ of the fluidized mass $0<\rho<0.8$ for $h_{i}=0,2,4,8$ normalized by the initial potential energy $E_{0}$ of the granular mass in the case $h_{i}=0$ as a function of time. The same symbols are used for the curves corresponding to the potential and kinetic energy and to the outline of the fluidized mass.

spreading. This could be due to the $2 \mathrm{D}$ geometry considered here.

[19] A very thin static layer of material on the plane $h_{i}=2$ (i.e., around 2 particles) significantly increases the mobility, i.e., runout distance, of the granular mass (Figure 1b). The runout distance is more than twice that calculated for $h_{i}=0$. However, the behavior of the mass is similar: the material spreads and stretches until a deposit is left on the plane with the same thickness $h_{s}$ of that obtained with $h_{i}=0$. For even larger thickness of the static layer $h_{i}=4$, the mobility increases and the shape of the flowing mass becomes rather different with a sharper front and a global triangular shape (Figure 1c). At the rear of the mass, the flow excavates the static layer as observed in the experiments. The granular mass propagates with a triangular shape with constant velocity (Figure 1c and inset in Figure 1b) as observed in the experiments over erodible bed. For $h=8$, a similar granular shape is created but now the avalanche propagates with increasing velocity (Figure 1d and inset in Figure 1b).

[20] As for a perturbation imposed on the top of a thin layer of static grains [Aranson et al., 2006], the flow of a granular material with dimensions much higher than the underlying static layer $\left(h_{0} \gg h_{i}\right)$ also generates soliton-like avalanches propagating with shape and velocity controlled by the depth of the substrate (inset in Figure 1b). Actually, the velocity is $75 \%$ higher at $t=100$ for the flow over an initial static layer $h_{i}=2$ than for the flow over a rigid bed at the same time and 3.6 times higher when $h_{i}=8$. As was observed in Figure 6 of Sovilla et al. [2006] for snow avalanches, the avalanche front velocity increases almost linearly with the depth of the erodible substrate $h_{i}$. Note that the velocities are larger than that observed in the 3D experiments of Pouliquen and Forterre [2002] especially in the early spreading phase. Similar high velocities at the early stage of the flow are reported by Sovilla et al. [2006, Figure 11] when using a depth-averaged model with entrainment.

\section{Static/Flowing Transition}

[21] The outline of the fluidized mass, corresponding to small values of $\rho(0<\rho<0.8)$, is represented in Figure 2 . The threshold $\rho=0.8$ has been chosen arbitrarily to visualize the interface between static and flowing material. The initially static material is fluidized (i.e., put into motion) instantaneously at the front of the flowing mass. Note that fluidization does not results from any imposed condition related to entrainment, but is simply calculated by the phase transition model for the order parameter. The avalanche "dives" into the erodible bed in a way that corresponds to the so-called frontal erosion described by Sovilla et al. [2006] when the snow cover is characterized by dry, low-density and cohesionless snow. Far from the front in the uphill direction, the so-called static/flowing interface is quasi-parallel to the inclined plane. A boundary layer with a thickness of a few grains between the bed and the flowing material is created near the bottom to link the static state at the base $\rho=1$ to the fluidized state in the upper layer where the calculated OP is $\rho \simeq 0.2$ (Figure $3 \mathrm{a}$ ). Interestingly enough, the position of this interface is almost independent of the initial thickness of the material lying on the plane. Entrainment seems to be controlled by the availability of the erodible mass, i.e., the potential energy of the whole system is only party consumed by entrainment. A critical value of entrainment, i.e., increase of the potential energy of the flowing material $(0<\rho<0.8)$ due to the increase of mass and therefore of the kinetic energy of the flowing mass, seems to be needed for generating traveling waves in order to balance the energy lost by friction (see inset in Figure 2).

[22] The vertical profiles of the order parameter at $x=$ 500 and $t=100$ actually show that $\rho(y)$ has an almost constant profile for $h_{i}=0,2,4$ and slightly differs for $h_{i}=$ 8 (Figure 3a). However, in the surface layer $\rho \simeq 0.23$ whatever $h_{i}$. On the contrary, near the free surface, the velocity significantly increases with the thickness of the
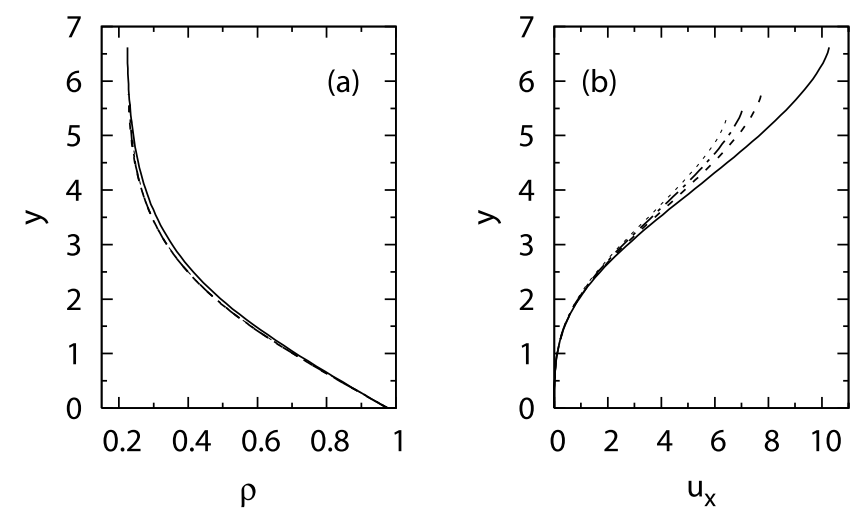

Figure 3. Vertical profile at $x=500$ and $t=100$ of (a) the order parameter $\rho$ and (b) the horizontal velocity $u_{x}$. The profiles are calculated for $h_{i}=0$ (dotted lines), $h_{i}=2$ (dashdotted lines), $h_{i}=4$ (dashed lines), and $h_{i}=8$ (full lines). 
initial static layer $h_{i}$ (Figure $3 \mathrm{~b}$ ). The velocity profiles obtained for different values of $h_{i}$ almost coincide in a basal boundary layer $h_{b} \leq 3$. Interestingly enough, $h_{b}$ almost corresponds to the thickness $h_{s}$ of the deposit left on top of the plane providing an interpretation of $h_{s}$. Numerical results suggest that traveling waves develop when the fluidized mass at the front is far enough from the basal boundary layer.

\section{Conclusion}

[23] In the simple case investigated here, entrainment of initially static material on the underlying slope is shown to significantly increase the mobility of avalanches. Most importantly, this study shows that even a very thin layer of particles lying on the solid bed could change its dynamics: for the thickness of the static layer below certain threshold, the avalanche decelerates and eventually stops, whereas for deeper layers, the avalanche transforms into a traveling wave and propagates along the slope without limit.

[24] The critical thickness for which the transition to traveling waves is observed obviously depends on the parameters used in the model, on the inclination of the plane and on the initial dimensions of the granular cap. Furthermore the simulations have been performed by assuming zero-velocity at the base of the flow. However the general mechanism of the transition can be understood in terms of the partial fluidization model. As the avalanche evolves, the "fluidization" diffuses into the static layer leading to its entrainment. The thicker boundary layer in turn leads to the reduction of friction losses (self-lubrication) and acceleration of the avalanche. Entrainment is shown here to depend essentially on the availability of mass lying on the slope (i.e., $h_{i}$ ). As the thickness of the erodible layer increases, more mass is included in the flow increasing its energy while eventually balancing the energy lost by friction. Nevertheless, more studies are needed to really understand the physical processes involved in this transition.

[25] The partial fluidization model not only provides insights into the static/flowing transition within the granular mass but allows to reproduce qualitatively granular flows over erodible bed when conventional depth-averaged model without entrainment fails [Pouliquen and Forterre, 2002]. As a result, taking into account partial fluidization of the granular mass appears to be a crucial ingredient of a successful theoretical description.

[26] Although the numerical experiments performed here are simple and therefore quite different from realistic geophysical situations, they provide some insights into the effect of an erodible bed on avalanche dynamics and on the generation of traveling waves (surges). As natural erodible beds are generally made of consolidated material, more energy would be required to put the static material into motion decreasing the efficiency of the process described here.

[27] Acknowledgments. We thank Shiva P. Pudasaini and an anonymous reviewer for their insightful comments. We thank Jean-Pierre Vilotte and Joe Goddard for fruitful discussions. This work was supported by ACI, CNRS and ANR, France (A.M. and F.B.) and by the U.S. Department of Energy, BES, grants DE-AC02-06CH11357 (I.A.) and DE-FG0204ER46135 (D.V. and L.T.). IPGP contribution 2276.

\section{References}

Aranson, I. S., and L. S. Tsimring (2002), Continuum theory of partially fluidized granular flows, Phys. Rev. E, 65, 061303.

Aranson, I. S., and L. S. Tsimring (2006), Patterns and collective behavior in granular media: Theoretical concepts, Rev. Mod. Phys., 78, 641-692.

Aranson, I. S., F. Malloggi, and E. Clement (2006), Transverse instability in granular flow down an incline, Phys. Rev. E, 73, 1-4, 050302(R).

Bouchut, F., E. D. Fernandez-Nieto, A. Mangeney, and P. Y. Lagree (2007), On new erosion models of Savage-Hutter type in avalanches, Acta Mech., in press.

Da Cruz, F., S. Emam, M. Prochnov, J. N. Roux, and F. Chevoir (2005), Rheophysics of dense granular materials: Discrete simulation of plane shear flows, Phys. Rev. E, 72, 021309.

Davies, T. R., and M. J. McSaveney (1999), Runout of dry granular avalanches, Can. Geotech. J., 36(2), 313-320.

Johnson, P. C., and R. Jackson (1987), Frictional-collisional constitutive relations for granular materials with application to plane shearing, J. Fluid Mech., 176, 67-93.

Landau, L. D., and E. M. Lifshitz (1980), Statistical Physics, Pergamon, New York.

Legros, F. (2002), The mobility of long-runout landslides, Eng. Geol., 63, $301-331$.

Lucas, A., and A. Mangeney (2007), Mobility and topographic effects for large Valles Marineris landslides on Mars, Geophys. Res. Lett., 34, L10201, doi:10.1029/2007GL029835.

Mallogi, F., J. Lanuza, B. Andreotti, and E. Clement (2006), Erosion waves: Transverse instabilities and fingering, Europhys. Lett., 75, 825.

Mangeney, A., F. Bouchut, N. Thomas, J. P. Vilotte, and M. O. Bristeau (2007), Numerical modeling of self-channeling granular flows and of their levee-channel deposits, J. Geophys. Res., 112, F02017, doi:10.1029/ 2006JF000469.

McDougall, S., and O. Hungr (2005), Dynamic modelling of entrainment in rapid landslides, Can. Geotech. J., 42, 1437-1448.

Pouliquen, O., and Y. Forterre (2002), Friction law for dense granular flows: application to the motion of a mass down a rough inclined plane, J. Fluid Mech., 453, 133-151.

Pudasaini, S. P., and K. Hutter (2007), Dynamics of Rapid Flows of Dense Granular Avalanches, Springer, New York.

Sovilla, B., P. Burlando, and P. Bartelt (2006), Field experiments and numerical modeling of mass entrainment in snow avalanches, J. Geophys. Res., 111, F03007, doi:10.1029/2005JF000391.

Volfson, D., L. Tsimring, and I. S. Aranson (2003), Partially fluidized shear granular flows: Continuum theory and molecular dynamics simulations, Phys. Rev. E, 68, 021301.

I. Aranson, Argonne National Laboratory, 9700 South Cases Avenue, Argonne, IL 60439, USA. (aronson@msd.anl.gov)

F. Bouchut, DMA, Ecole Normale Supérieure, 45 rue d'Ulm, F-75230 Paris cedex 05, France. (fbouchut@dma.ens.fr)

A. Mangeney, L. Tsimring, and D. Volfson, Institute for Nonlinear Science, University of California San Diego, 9500 Gilman Drive, La Jolla, CA 92093-0402, USA. (amangeney@ucsd.edu; 1tsimring@ucsd.edu; dvolfson@ucsd.edu) 